\title{
Psychological Predictors of Health Anxiety in Response to the Zika Virus
}

\author{
Shannon M. Blakey ${ }^{1} \cdot J^{\prime}$ onathan S. Abramowitz ${ }^{1}$
}

Published online: 23 October 2017

(C) Springer Science+Business Media, LLC 2017

\begin{abstract}
The threat of a United States (U.S.) Zika virus pandemic during 2015-2016 was associated with public anxiety. Such threats represent opportunities to examine hypotheses about health anxiety. The present study investigated psychological predictors of Zika-related anxiety during the 2015-2016 outbreak. U.S. adults $(N=216)$ completed a battery of measures assessing Zika-related anxiety as well as psychological variables hypothesized to predict anxious responding to the threat of a domestic Zika outbreak. Contrary to hypotheses, regression analyses indicated that only contamination severity overestimates and greater Zika knowledge significantly predicted Zika-related anxiety. Study limitations and clinical implications are discussed.
\end{abstract}

Keywords Health anxiety · Contamination $\cdot$ Zika

\section{Introduction}

Originally discovered in 1947 in the Zika Forest of Uganda, the Zika virus is usually transmitted to humans via a bite from an infected mosquito; there also have been reports of sexually transmitted Zika cases (Hills, Russell, Hennessey, Williams, Oster, Fischer, \& Mead, 2016). Although there is no vaccine or treatment for Zika virus disease, mortality rates are extremely low, and not all infected individuals display or even notice their symptoms. The most common symptoms of Zika are fever, rash, joint pain, and

Shannon M. Blakey

sblakey@unc.edu

1 Department of Psychology and Neuroscience, The University of North Carolina at Chapel Hill, CB \# 3270, Davie Hall, Chapel Hill, NC 27599, USA conjunctivitis, with symptoms usually mild in severity and lasting 3-7 days (Centers for Disease Control and Prevention [CDC], 2016). However, Zika has been linked to increased risk for Guillain-Barré syndrome as well as microcephaly among children born to mothers infected with the virus during pregnancy (Deckard et al., 2016).

The confirmed human Zika virus case in Brazil in May 2015 commenced the most recent (and ongoing, at the time of writing) international outbreak, which was declared a Public Health Emergency by the World Health Organization (WHO) in February 2016. As of June 2016, 61 countries and territories reported active mosquito-borne transmission of the Zika virus, with the majority of global cases being documented throughout Latin America (WHO, 2016). Extensive news and media coverage of the Zika outbreak was followed by increased fear in the U.S. that the Zika virus might become a domestic pandemic. Despite public statements from the National Institute of Allergy and Infectious Diseases assuring the public that risk of a major U.S. outbreak is low, concern among U.S. residents remains high (Santora, 2016).

Health anxiety-a construct which refers to the inappropriate or exaggerated concern about one's health status relative to one's actual health-is conceptually relevant to a number of psychological conditions, including obsessive compulsive disorder (OCD), illness anxiety disorder, somatic symptom disorder, and other anxiety disorders (e.g., Taylor, McKay, \& Abramowitz, 2012). Cognitive-behavioral models of health anxiety posit that distress arises as a result of mistaken beliefs about illness, health, and the meaning of ambiguous body sensations (e.g., Abramowitz \& Braddock, 2011; Taylor \& Asmundson, 2004; Warwick, 1989). Specifically, threatening appraisals of somatic sensations (e.g., "if I feel feverish, I might have Zika") trigger anxiety and worry, as well as urges to perform precautionary (i.e., safety) 
behaviors such as decontamination, reassurance-seeking, or avoidance of potential contaminants (e.g., Dorfan \& Woody, 2011). Although these behaviors often reduce health-related anxiety in the short term, such relief is typically fleeting. Moreover, such behaviors have been shown to maintain and even exacerbate health anxiety for a long term (e.g., Deacon \& Maack, 2008; Gangemi, Mancini, \& van den Hout, 2012; Olatunji, Etzel, Tomarken, Ciesielski, \& Deacon, 2011).

Theoretical models of health anxiety implicate additional psychological mechanisms in its development and maintenance. For instance, overestimates of the likelihood and severity of illness are thought to drive health anxiety (e.g., Abramowitz \& Braddock, 2011; Becker \& Janz, 1984; Taylor \& Asmundson, 2004). Previous studies showing that overestimates of threat predict anxious responding to disease outbreaks of SARS, swine (H1N1) flu, avian (H5N1) flu, and the Ebola virus offer empirical support for this hypothesis (e.g., Bish \& Michie, 2010; Blakey, Reuman, Jacoby, \& Abramowitz, 2015; Lau, Kim, Tsui, \& Griffiths, 2008; Wheaton, Abramowitz, Berman, Fabricant, \& Olatunji, 2012; Xie, Stone, Zheng, \& Zhang, 2011).

Anxiety sensitivity and body vigilance are additional cognitive biases involved in the development and maintenance of health anxiety. Anxiety sensitivity refers to the propensity to misinterpret anxious arousal (or other ambiguous physical sensations) as dangerous (e.g., Taylor, Zvolensky, Cox, Deacon, Heimberg, Ledley, 2007). Individuals high in anxiety sensitivity are prone to self-monitor for unexplained body sensations (e.g., a hot flash) in an attempt to quickly detect and respond to potential threat. Indeed, previous research shows that anxiety sensitivity and body vigilance predict anxious responding to health status in general (e.g., Gerolimatos \& Edelstein, 2012; Krautwurst, Gerlach, Gomille, Hiller, \& Witthöff, 2014; Schmidt, Lerew, \& Trakowski, 1997), as well as to specific disease outbreaks (e.g., Blakey et al., 2015; Wheaton et al., 2012; Xie et al., 2011). It follows that someone who believes unexplained body sensations forecast medical catastrophe would continually monitor for bodily changes, detect them rapidly, misconstrue them as threatening (i.e., a symptom of Zika), and consequently experience anxiety.

Disgust sensitivity, the tendency to experience disgust across multiple domains, also may predict Zika-related anxiety. In light of research demonstrating that disgust responding features prominently in contamination fear (Cisler, Brady, Olatunji, \& Lohr, 2010; Olatunji \& Sawchuck, 2005; Tolin, Woods, \& Abramowitz, 2006), general health anxiety (e.g., Brady, Cisler, \& Lohr, 2014; Davey \& Bond, 2006; Fan \& Olatunji, 2013; Olatunji, 2009), hypochondriasis (e.g., Davey \& Bond, 2006; Weck, Esch, \& Rohrmann, 2014), and other disease outbreak concerns (Blakey et al., 2015; Brand, McKay, Wheaton, \& Abramowitz, 2013), it stands to reason that disgust sensitivity is also related to anxiety over Zika. General distress also has been associated with illness anxiety (e.g., Niles et al., 2014; Scott et al., 2007) and is another candidate predictor of Zika anxiety, perhaps because general distress perpetuates the types of negative interpretive biases that are involved in health anxiety (e.g., Reif, Hiller, \& Margraf 1998).

Research shows that publicizing disease outbreaks can engender health anxiety even among the medically healthy (e.g., Taylor \& Asmundson, 2004). That is, although dissemination of information related to diseases and recommended hygiene behaviors may mitigate the spread of disease, excessive (or sensationalized) media coverage might also promote exaggerated threat estimates regarding the likelihood and/or severity of contracting a feared disease among those vulnerable to developing clinically severe health anxiety. Correlational research shows positive associations between media coverage and the public's anxiety related to epidemics such as SARS (Xie et al., 2011) and Ebola (e.g., Cheung, 2015). Cognitive interventions for health anxiety emphasize the therapeutic effects of corrective information (e.g., Clark, 1986); therefore, one might predict that individuals possessing more accurate knowledge about the Zika virus and its outbreak would report less Zika-related anxiety.

Results from research examining psychological factors related to health anxiety in response to discrete outbreaks are mixed. Such inconsistent findings might be due to diverse study methodology or sample idiosyncrasies. Another consideration is that the diseases studied previously (e.g., Avian flu, swine flu, cholera, Ebola) are qualitatively distinct. For example, whereas the Zika virus and Avian flu are primarily transmitted to humans via animals, a person may get cholera by drinking contaminated water, yet contract swine flu or Ebola through contact with human bodily fluids. Such meaningful differences between previously studied diseases (e.g., method of disease transmission) may have influenced the detected relationships between psychological constructs highlighted in etiological models and disease outbreak anxiety (e.g., Liao, Cowling, Lam, \& Fielding, 2011).

Research examining the psychological predictors of anxiety in response to disease outbreak is limited, yet understanding these processes may inform the treatment and prevention of health anxiety (Bish \& Michie, 2010), which is associated with increased (and unnecessary) healthcare costs and reduced quality of life (e.g., missed days at work; Taylor $\&$ Asmundson, 2004). Furthermore, continued research in this area is needed to test the replicability and generalizability of previous findings to new participant samples and disease contexts. By investigating the consistency of psychological factors that may contribute to outbreak-related public distress, psychological models that seek to explain clinically significant health anxiety may be refined and updated. Clarifying the factors that contribute to health anxiety in the wake of global pandemics also may help clinicians identify 
individuals who might be vulnerable to exacerbated health anxiety-related symptoms (e.g., a patient with OCD who becomes increasingly distressed about contamination during an illness outbreak).

The present study was designed to better understand the psychological factors associated with Zika-related anxiety in a sample of adults residing in the U.S. As discussed above, the extant literature implicates several candidate predictors of anxious responding to the threat of a U.S. Zika virus pandemic. Accordingly, we hypothesized that after controlling for age and gender, less factual knowledge, but greater levels of anxiety sensitivity, body vigilance, contamination-related threat overestimations, general distress, disgust sensitivity, and general health anxiety would predict greater Zikarelated anxiety.

\section{Design}

\section{Participants}

Two hundred sixteen affiliates (i.e., students, faculty, staff, and other employees) at a large university in the southeastern U.S. volunteered to participate in this study (i.e., participants were not compensated). The study was advertised through a department research participation pool as well as a listserv that publicized psychology research opportunities. The sample was mostly female $(n=159 ; 77.2 \%)$, with a mean age of 34.18 years $(S D=17.05$, range was 18 to 73$)$. Less than half of the sample self-identified as a current undergraduate student $(n=95 ; 46.1 \%)$. The majority of participants identified as white $(n=168 ; 81.6 \%)$, with $6.8 \%$ identifying as Asian $(n=14), 6.3 \%$ identifying as African American or black ( $n=13)$, and $5.3 \%$ identifying with another racial/ethnic group $(n=11)$

\section{Procedure}

Data were collected from February 4th through March 23rd, 2016, during which time Zika received substantial media attention. Participants were directed to a survey link hosted by Qualtrics, a secure online survey development tool. Participants completed the measures described below in randomized order, followed by a demographics questionnaire. Two distractor items (e.g., "please answer Always True for this item") were also included among the measures to increase the probability that only valid responses from attentive participants would be included in analyses (Meade \& Craig, 2012). This study was approved by the university's Institutional Review Board, and informed consent was obtained from all individual participants included in the study.

\section{Main Outcome Measure}

\section{Zika Anxiety Inventory (ZAI)}

The ZAI is a nine-item measure designed to assess anxiety associated with the Zika virus. Typical ZAI items are "To what extent are you concerned about the Zika virus?" and "How concerned are you that someone you know could become infected with Zika?" Items are rated from $1=$ not at all to $5=$ very much and were inspired by those used in studies assessing swine flu and Ebola virus anxiety (Blakey et al., 2015; Wheaton et al., 2012). Possible scores range from 9 to 45, with lower scores indicating lower Zika-related anxiety.

Item analyses were conducted according to guidelines set forth by DeVellis (1991) to assess the ZAI's reliability and suitability for further analyses. No items had corrected total-item correlations falling below the acceptable level of 0.30 (Nunnally \& Bernstein, 1994). Further, total scale reliability indices (Cronbach's $\alpha$ ) were comparable following deletion of any item (i.e., the scale's internal reliability was not substantially improved by the deletion of any item). The ZAI demonstrated good internal consistency $(\alpha=0.84)$ in the current sample. The distribution of scores on the ZAI was also free of significant skew (0.78) and kurtosis (0.10).

\section{Predictor Measures}

\section{Anxiety Sensitivity Index-3 (ASI-3; Taylor et al., 2007)}

The ASI-3 (derived from the original ASI; Reiss, Peterson, Taylor, Schmidt, \& Weems, 2008) is an 18-item measure of beliefs regarding the dangerousness of anxiety along physical (e.g., "it scares me when my heart beats rapidly"), cognitive (e.g., "it scares me when I am unable to keep my mind on a task"), and social (e.g., "it scares me when I blush in front of other people") domains. Participants rate their agreement with these statements on a $0=$ very little to $4=$ very much scale. Possible subscale scores range from 0 to 24 , and potential total scores range from 0 to 72 , with lower scores indicating lower anxiety sensitivity. The ASI-3 has demonstrated a three-factor structure with good reliability and criterion-related validity in previous research (Taylor et al., 2007). The ASI-3 physical, cognitive, and social concern subscales showed good to excellent internal consistency in the current sample $(\alpha \mathrm{s}=0.87,0.90$, and 0.80 , respectively).

\section{Body Vigilance Scale (BVS; Schmidt et al., 1997)}

The BVS is a four-item measure of one's tendency to attend to anxiety-related body sensations. The first three items assess attentional focus to, sensitivity to changes in, and 
amount of time devoted to monitoring body sensations on a $0=$ not at all to $10=$ extremely scale. One of these items is "I am very sensitive to changes in my internal body sensations." In the fourth item, respondents separately rate the degree of attention paid to 15 body sensations (e.g., heart rate) on a $0=$ none to $10=$ extreme scale, which are averaged to yield a single item score. Possible scores range from 0 to 40 , with lower scores indicating lower body vigilance. The BVS has shown good internal consistency and test-retest reliability in previous research (Schmidt et al., 1997). The BVS showed excellent internal consistency $(\alpha=0.94)$ in the current sample.

\section{Contamination Cognitions Scale (CCS; Deacon \& Maack, 2008)}

The CCS assesses one's tendency to overestimate the likelihood and severity of contamination from a variety of commonplace objects (e.g., money). Participants separately rate the likelihood and severity of contamination for each item on a $0=$ not at all to $100=$ extremely scale. Separate likelihood (CCS-L) and severity (CCS-S) subscales are formed by computing the average response for items falling on the CCS-L and CCS-S subscales, respectively. Possible subscale scores range from 0 to 100 , with lower scores indicating lower likelihood and severity estimates. The internal consistency was excellent for the CCS-L $(\alpha=0.95)$ and CCS-S $(\alpha=0.96)$ in the current sample.

\section{Depression Anxiety Stress Scales-21 (DASS-21; Antony, Bieling, Cox, Enns, \& Swinson, 1998)}

The DASS-21 is a short-form version of the 42-item DASS (Lovibond \& Lovibond, 1995) that assesses subjective distress over the past week along three subscales: depression, anxiety, and stress. Participants rate how each of the 21 statements (e.g., "I felt downhearted and blue") apply to them on a $0=$ rarely to $4=$ very much, or most of the time scale. Possible scores range from 0 to 126 (raw scores are multiplied by a factor of two to yield total scores), with lower scores indicating lower subjective distress. The DASS21 has demonstrated good reliability and construct validity in clinical and nonclinical samples (Henry \& Crawford, 2005). The DASS-21 showed excellent internal consistency $(\alpha=0.93)$ in the current sample.

\section{Disgust Scale-Revised (DS-R; Olatunji et al., 2007)}

The DS-R, revised from the original DS (Haidt, McCauley, \& Rozin, 1994), is a 25 -item measure of respondents' propensity to experience disgust across multiple domains. Participants rate the degree to which they might find a number of scenarios (e.g., "you see maggots on a piece of meat in an outdoor garbage pail") disgusting on a $0=$ strongly disagree to $4=$ strongly agree scale. Ratings are averaged across items and the total score ranges from 0 to 4 , with lower scores indicating lower disgust sensitivity. The DS-R has demonstrated adequate internal consistency and convergent validity in previous work (Olatunji et al., 2007) and showed good internal consistency $(\alpha=.86)$ in the current sample.

Short Health Anxiety Inventory (SHAI; Salkovskis, Rimes, Warwick, \& Clark, 2002)

The SHAI is an 18-item self-report measure of general health anxiety. Items measure worry about health, awareness of bodily sensations or changes, and feared consequences of having an illness using a multiple choice format with changing anchors that use a 0 to 3 scale. Two typical items are " 0 - I do not worry about my health; 1 -I occasionally worry about my health; 2 -I spend much of my time worrying about my health; 3 -I spend most of my time worrying about my health"; and " 0 - I do not have any difficulty taking my mind off thoughts about my health; $1-\mathrm{I}$ sometimes have difficulty taking my mind off thoughts about my health; 2-I often have difficulty taking my mind off thoughts about my health; 3-Nothing can take my mind off thoughts about my health." Possible scores range from 0 to 54 , with lower scores indicating lower general health anxiety. The SHAI has demonstrated good reliability and validity as a measure of health anxiety in clinical and nonclinical samples (Abramowitz et al., 2007; Salkovskis et al., 2002). The SHAI showed good internal consistency $(\alpha=0.85)$ in the current sample.

\section{Zika Facts Quiz (ZFQ)}

The ZFQ is an eight-item multiple choice measure of knowledge about the Zika virus and 2015-2016 global outbreak. Participant responses are scored on a $0=$ incorrect to $1=$ correct coding scheme; possible scores range from 0 to 8 , with higher scores indicating greater knowledge about the Zika virus and 2015-2016 outbreak. Quiz items were inspired by the Ebola Facts Quiz used by Blakey et al., (2015) based on information published by the CDC on their Zika Virus webpage (CDC, 2016).

\section{Results}

\section{Data Screening}

Data collected from the 216 respondents who passed the distractor items were screened to assess concordance with statistical assumptions. Distributions of scores on all of the study measures were free of significant skew (all 
values $<3$ ) and kurtosis (all values $<5$ ). No univariate outliers were detected, but ten multivariate outliers were noted (Mahalanobis distances fell beyond critical $\chi_{\mathrm{df}}^{2}=11$ value of 31.264). These multivariate outlier cases were excluded from further analyses due to the possible bias of regression point estimates and sufficiently large sample. Score distributions of the remaining 206 participants showed no significant skew, kurtosis, univariate outlier indices, or multivariate outlier indices (see Table 1).

\section{Descriptive Statistics}

As would be expected in a nonclinical sample, our participants reported experiencing relatively mild Zika-related anxiety, on average. Scores on predictor measures fell within the typical range for nonclinical samples. Finally, Table 1 shows that participants had a variable degree of factual knowledge about the Zika virus, with ZFQ scores approximating a normal distribution around an average 69\% accuracy.

\section{Bivariate Correlations}

Two-tailed bivariate correlations were conducted to examine the relation between Zika virus concerns and other study variables. As seen in Table 2, there were small to moderately positive correlations between the ZAI and all study variables except for the DASS-21, age, and gender.

\section{Regression Analyses Predicting Zika Anxiety}

A simultaneous linear regression was conducted to determine which psychological variables independently predicted
Table 1 Study measure descriptive statistics

\begin{tabular}{lrllllc}
\hline Measure & $M$ & $(\mathrm{SD})$ & Min & Max & Skew & Kurtosis \\
\hline Zika anxiety inventory & 6.90 & $(5.00)$ & 0 & 23 & 0.78 & 0.10 \\
Anxiety sensitivity inventory-3 physical concerns & 4.42 & $(4.52)$ & 0 & 24 & 1.68 & 3.01 \\
Anxiety sensitivity inventory-3 cognitive concerns & 2.56 & $(2.56)$ & 0 & 19 & 2.12 & 4.73 \\
Anxiety sensitivity inventory-3 social concerns & 7.20 & $(7.20)$ & 0 & 23 & 0.74 & 0.15 \\
Body vigilance scale & 15.88 & $(7.14)$ & 0 & 32 & 0.02 & -0.55 \\
Contamination cognitions scale-likelihood estimates & 38.78 & $(23.62)$ & 0 & 100 & 0.32 & -0.68 \\
Contamination cognitions scale-severity estimates & 36.26 & $(22.42)$ & 0 & 100 & 0.62 & -0.06 \\
Depression anxiety stress scales-21 & 19.65 & $(17.32)$ & 0 & 92 & 1.64 & 2.91 \\
Disgust scale-revised & 1.97 & $(0.56)$ & 0.64 & 3.52 & 0.17 & -0.25 \\
Short health anxiety inventory & 12.44 & $(5.75)$ & 1 & 31 & 0.60 & 0.28 \\
Zika facts quiz & 5.55 & $(1.60)$ & 0 & 8 & -0.80 & 0.30 \\
\hline
\end{tabular}

Table 2 Bivariate correlations between study measures

\begin{tabular}{|c|c|c|c|c|c|c|c|c|c|c|c|c|}
\hline & ZAI & ASI-P & ASI-C & ASI-S & BVS & CCS-L & CCS-S & DASS-21 & DS-R & SHAI & ZFQ & Age \\
\hline ASI-P & $0.28 * *$ & - & & & & & & & & & & \\
\hline ASI-C & $0.24 * *$ & $0.46^{* *}$ & - & & & & & & & & & \\
\hline ASI-S & $0.22 * *$ & $0.47 * *$ & $0.57 * *$ & - & & & & & & & & \\
\hline BVS & $0.29 * *$ & $0.45^{* *}$ & $0.39 * *$ & $0.36 * *$ & - & & & & & & & \\
\hline CCS-L & $0.22 * *$ & $0.23 * *$ & 0.09 & $0.18^{*}$ & $0.26 * *$ & - & & & & & & \\
\hline CCS-S & $0.26 * *$ & $0.16^{*}$ & 0.12 & 0.11 & $0.22 * *$ & $0.53 * *$ & - & & & & & \\
\hline DASS-21 & 0.14 & $0.32 * *$ & $0.56 * *$ & $0.51 * *$ & $0.26^{* *}$ & 0.12 & -0.01 & - & & & & \\
\hline DS-R & $0.22 * *$ & $0.32 * *$ & $0.26 * *$ & $0.27 * *$ & $0.19 *$ & $0.30 * *$ & $0.35 * *$ & $-0.22 * *$ & - & & & \\
\hline SHAI & $0.30 * *$ & $0.52 * *$ & $0.48 * *$ & $0.46^{* *}$ & $0.57 * *$ & $0.19^{*}$ & 0.12 & $0.45^{* *}$ & $0.27 * *$ & - & & \\
\hline ZFQ & $0.15^{*}$ & -0.06 & $-0.18^{*}$ & -0.08 & 0.02 & -0.08 & $-0.17 *$ & -0.12 & $-0.25^{* *}$ & $<0.01$ & - & \\
\hline Age & 0.01 & -0.10 & $-0.20 *$ & $-0.26^{* *}$ & -0.06 & $-0.23 * *$ & $-0.24 * *$ & $-0.24 * *$ & $-0.35^{* *}$ & $-0.18 *$ & $0.36^{* *}$ & - \\
\hline Gender & -0.07 & -0.07 & -0.02 & -0.01 & -0.01 & -0.10 & -0.06 & -0.02 & $-0.17^{*}$ & -0.06 & -0.08 & -0.05 \\
\hline
\end{tabular}

ZAI Zika anxiety inventory, ASI-P anxiety sensitivity inventory-3 physical concerns subscale, $A S I-C$ anxiety sensitivity inventory-3 cognitive concerns subscale, $A S I-S$ anxiety sensitivity inventory-3 social concerns subscale, $B V S$ body vigilance scale, $C C S-L$ contamination cognitions scale-likelihood, $C C S$-S contamination cognitions scale-severity, DASS-21 depression anxiety stress scales-21, DS-R disgust scale-revised, SHAI short health anxiety inventory, ZFQ Zika facts quiz, gender was coded 0 - female and 1 -male in all analyses reported

$* p<.05$

$* * p<.005$ 
Zika anxiety (see Table 3). The overall model was significant and accounted for approximately $21.3 \%$ of variance in $\mathrm{ZAI}$ scores, $F(12,191)=4.39, p<.001$. Within the full model, only CCS-S $(p<.05)$ and ZFQ $(p<.01)$ scores emerged as significant unique predictors of Zika virus anxiety. Specifically, concerns about the severity of contamination uniquely accounted for $2.5 \%$ of variability in ZAI scores and Zika knowledge accounted for $3.2 \%$ of variability in ZAI scores. Coefficient estimates for the CCS-S and ZFQ were both positive, indicating that greater contamination severity overestimates and Zika knowledge predicted greater Zika anxiety. No other variables emerged as uniquely significant predictors of Zika anxiety in the current sample (all $p \mathrm{~s} \geq .15$ ).

\section{Discussion}

News of the 2015-2016 Zika outbreak has received substantial attention from public health organizations as well as the popular media. There is also growing concern that travelers coming to the U.S. after visiting Zika-infected regions of the world might increase the chance of a domestic pandemic. Underscoring the severity of fear of Zika in the U.S., in April 2016, the U.S. government reallocated $\$ 510$ million in funds that originally were dedicated to battling the Ebola virus, and redirected the funds toward containment of the Zika virus (McNeil, 2016). The present study was designed to identify which psychological factors predict health anxiety in response to the threat of a domestic Zika virus pandemic. Results partially supported our hypothesis; only contamination likelihood overestimates and factual knowledge emerged as significant unique predictors of Zika-related anxiety.

Our finding that contamination-related threat estimates significantly predicted Zika-related anxiety is consistent with previous research on anxious responding to the Ebola virus (Blakey et al., 2015), avian flu (e.g., Lau et al.,
2008), and swine flu (e.g., Wheaton et al., 2012). However, whereas Blakey et al., (2015) found that likelihood overestimates were related to Ebola anxiety, findings from the present study highlight the unique relation between severity overestimates and Zika anxiety. It is possible that differences between diseases (e.g., in symptom profile, means of transmission, etc.) determine which predictor variables have greatest relevance for outbreak-specific health anxiety. Thus, diseases such as avian flu, swine flu, Ebola virus, and Zika virus may each generate somewhat different patterns of health anxiety. For example, whereas the Ebola virus was associated with low probability of contraction (for U.S. residents) and high symptom severity, the odds of a U.S. resident contracting Zika (the symptoms of which are generally mild and brief) were much higher during the time of data collection. Future studies of health anxiety specific to discrete pandemics should assess for beliefs about the outbreak (e.g., regarding contagion) in addition to factual knowledge about the disease itself.

Results showed that more accurate knowledge about the Zika virus was associated with greater Zika anxiety. This finding was unexpected and highlights that informationseeking may be either adaptive (i.e., corrective informationgathering) or maladaptive (e.g., reassurance-seeking). Given the cross-sectional nature of our study, it is possible that some fearful participants coped with their Zika anxiety by seeking out knowledge and information about the disease, thereby obfuscating our hypothesized effect. In light of previous studies showing that information-searching (i.e., cyberchondria; Fergus, 2013) increases dramatically in the initial weeks after disease outbreak announcements are made (Tausczik, Faasse, Pennebaker, \& Petrie, 2012), longitudinal research examining the relation between health anxiety and disease knowledge over time would be helpful. Future research also should consider whether one's perceived versus one's actual knowledge best predicts anxious responding
Table 3 Simultaneous linear regression predicting Zika anxiety

\begin{tabular}{lrrrrrr}
\hline Variable & $\mathrm{B}$ & $\mathrm{SE}_{\mathrm{B}}$ & $\beta$ & \multicolumn{1}{c}{$t$} & \multicolumn{1}{c}{$p$} & \multicolumn{2}{c}{$\mathrm{spr}^{2}$} \\
\hline Anxiety sensitivity inventory-3 physical concerns & 0.08 & 0.09 & 0.07 & 0.86 & 0.390 & 0.003 \\
Anxiety sensitivity inventory-3 cognitive concerns & 0.16 & 0.12 & 0.12 & 1.33 & 0.185 & 0.007 \\
Anxiety sensitivity inventory-3 social concerns & 0.03 & 0.09 & 0.03 & 0.30 & 0.762 & $<0.001$ \\
Body vigilance scale & 0.03 & 0.06 & 0.05 & 0.59 & 0.557 & 0.001 \\
Contamination cognitions scale-likelihood estimates & 0.01 & 0.02 & 0.05 & 0.64 & 0.520 & 0.002 \\
Contamination cognitions scale-severity estimates & 0.04 & 0.02 & 0.20 & 2.45 & 0.015 & 0.025 \\
Depression anxiety stress scales-21 & -0.01 & 0.02 & -0.02 & -0.22 & 0.826 & $<0.001$ \\
Disgust scale-revised & 0.98 & 0.69 & 0.11 & 1.42 & 0.157 & 0.008 \\
Short health anxiety inventory & 0.11 & 0.08 & 0.13 & 1.42 & 0.158 & 0.008 \\
Zika facts quiz & 0.62 & 0.22 & 0.20 & 2.81 & 0.005 & 0.032 \\
Age & 0.03 & 0.02 & 0.09 & 1.18 & 0.239 & 0.006 \\
Gender & -0.01 & 0.78 & 0.00 & -0.01 & 0.992 & $<0.001$ \\
\hline
\end{tabular}

$s p r^{2}$-squared semipartial correlation 
to the threat of a disease outbreak. The possibility that perception of one's own knowledge is more strongly related to health anxiety than is one's own actual knowledge is consistent with previous research. Also related to this possibility is literature implicating the role of intolerance of uncertainty in the experience of health anxiety, i.e., the tendency to consider it unacceptable that a negative event could occur at all, however small its actual probability may be (e.g., Dugas Dugas, Gosselin, \& Ladouceur, 2001; Fetzner, Asmundson, Carey, Thibodeau, Brandt, Zvolensky, \& Carleton, 2014; Rosen \& Knäuper, 2009; Xie et al., 2011).

Contrary to our hypothesis, neither general distress, general health anxiety, anxiety sensitivity domains, body vigilance, nor disgust sensitivity emerged as uniquely significant predictors of Zika-related anxiety. Although this could merely be an artifact of our sample's idiosyncrasies or demographic makeup, these findings also might be interpreted to suggest that Zika fears are not driven by the same psychological factors that engender more generalized health anxiety or related conditions (e.g., OCD, illness anxiety disorder). The possibility that the cognitive-behavioral model of generalized health anxiety may not apply to fearful responding to discrete illness outbreaks is bolstered by the fact that only a few purported psychological predictors of health anxiety have been linked to anxious responding to outbreaks other than the Zika virus, i.e., outbreaks such as those involving avian flu (Liao et al., 2011), swine flu (Wheaton et al., 2012), and SARS (Xie et al., 2011). Our finding that generalized health anxiety was correlated with, but not predictive of, Zika anxiety is also interesting and suggests that anxious responding in the wake of discrete outbreaks is related to, yet distinct from, more generalized health anxiety. Future research should explore the degree to which certain psychological factors influence health anxiety in general as compared to health anxiety in response to specific illnesses in both clinical and nonclinical samples.

This study's findings are subject to a number of limitations. First, we recruited an educated, nonclinical sample that was not nationally representative; therefore, our data do not speak to individuals with clinical levels of health anxiety, residents of high-incidence areas (e.g., Latin Americans), or individuals from more diverse backgrounds. Similarly, although our sample included mostly women around childbearing age, we did not assess for current or planned pregnancy or changes in sexual activity as a result of the Zika outbreak; therefore, our data do not speak to the intensity or burden of Zika-related anxiety on prospective parents in particular. Nevertheless, findings from this study may still inform prevention and treatment interventions for clinically significant health anxiety (or related conditions such as OCD) among otherwise healthy or low-risk individuals. Future research might also include variables not assessed in the present investigation, such as the intolerance of uncertainty (e.g., Fetzner et al., 2014; Rosen \& Knäuper, 2009), beliefs related to coping ability (e.g., Salkovskis \& Warwick, 2001), beliefs about the inadequacy of medical services for treating illness (e.g., Salkovskis \& Warwick, 2001), and metacognitive beliefs (e.g., Bailey \& Wells, 2015).

Another limitation is that all data were obtained via selfreport, which might have inflated the associations among study variables. Similarly, the cross-sectional design of this study precludes drawing conclusions regarding causality or temporal precedence. For instance, it is possible that individuals who overestimate the probability of becoming contaminated are more prone to be afraid of the threat of the Zika virus; alternatively, individuals experiencing Zika-related anxiety may come to believe that contamination was more likely than they had previously thought. Additional research utilizing a longitudinal and multimethod assessment design is needed to determine the risk factors of experiencing health anxiety in response to discrete disease epidemics.

\section{Compliance with Ethical Standards}

Conflict of interest Shannon M. Blakey and Jonathan S. Abramowitz declare that they have no conflict of interest.

Human and Animal Rights and Informed Consent All procedures performed in studies involving human participants were in accordance with the ethical standards of the institutional and/or national research committee and with the 1964 Helsinki declaration and its later amendments or comparable ethical standards. Informed consent was obtained from all individual participants included in the study. This article does not contain any studies with animals performed by the authors.

\section{References}

Abramowitz, J. S., \& Braddock, A. E. (2011). Hypochondriasis and health anxiety. Cambridge, MA: Hogrefe \& Huber.

Abramowitz, J.S., Olatunji, B.O., \& Deacon, B.J. (2007). Health anxiety, hypochondriasis, and the anxiety disorders. Behavior Therapy, 38, 86-94. doi:10.1016/j.beth.2006.05.001

Antony, M. M., Bieling, P. J., Cox, B. J., Enns, M. W., \& Swinson, R. P. (1998). Psychometric properties of the 42-item and 21-item versions of the Depression Anxiety Stress Scales (DASS) in clinical groups and a community sample. Psychological Assessment, $10,176-181$.

Bailey, R., \& Wells, A. (2015). The contribution of metacognitive beliefs and dysfunctional illness beliefs in predicting health anxiety: An evaluation of the metacognitive versus the cognitive models. Clinical Psychologist, 20, 129-137.

Becker, M. H., \& Janz, N. K. (1984). The Health Belief Model: A decade later. Health Education Quarterly, 11, 1-47. doi:10.1177/109019818401100101.

Bish, A., \& Michie, S. (2010). Demographic and attitudinal determinants of protective behaviors during a pandemic: A review. British Journal of Health Psychology, 15, 797-824.

Blakey, S. M., Reuman, L., Jacoby, R. J., \& Abramowitz, J. S. (2015). Tracing "Fearbola": Psychological predictors of 
anxious responding to the threat of Ebola. Cognitive Therapy and Research, 39, 816-825.

Brady, R. E., Cisler, J. M., \& Lohr, J. M. (2014). Specific and differential prediction of health anxiety by disgust sensitivity and propensity. Anxiety, Stress, and Coping, 27, 90-99.

Brand, J., McKay, D., Wheaton, M. G., \& Abramowitz, J. S. (2013). The relationship between obsessive compulsive beliefs and symptoms, anxiety and disgust sensitivity, and Swine Flu fears. Journal of Obsessive-Compulsive and Related Disorders, 2, 200-206.

Center for Disease Control and Prevention (CDC) (2016). About Zika virus disease. Retrieved from http://www.cdc.gov/zika/about/ index.html.

Cheung, E. Y. L. (2015). An outbreak of fear, rumours, and stigma: Psychosocial support for the Ebola Virus Disease outbreak in West Africa. Intervention, 13, 45-84.

Cisler, J. M., Brady, R. E., Olatunji, B. O., \& Lohr, J. M. (2010). Disgust and obsessive beliefs in contamination-related OCD. Cognitive Therapy and Research, 34, 439-448.

Clark, D. M. (1986). A cognitive approach to panic. Behaviour Research and Therapy, 24, 461-470.

Davey, G. L., \& Bond, N. (2006). Using controlled comparisons in disgust psychopathology research: The case of disgust, hypochondriasis and health anxiety. Journal of Behavior Therapy and Experimental Psychiatry, 37, 4-15.

Deacon, B. J., \& Maack, D. J. (2008). The effects of safety behaviors on the fear of contamination: An experimental investigation. Behaviour Research and Therapy, 46, 537-547.

Deckard, D. T., Chung,. M., Books, J. T., Smith, J. C., Woldai, S., Hennessey, M., ... Mead, P. (2016). Male-to-male sexual transmission of Zika virus-Texas, January 2016. Morbidity and Mortality Weekly Report (MMWR), 65, pp. 372-374.

DeVellis, R. F. (1991). Scale development: Theory and applications. Newbury Park: Sage.

Dorfan, N. M., \& Woody, S. R. (2011). Danger appraisals as prospective predictors of disgust and avoidance of contaminants. Journal of Social and Clinical Psychology, 30, 105-122.

Dugas, M. J., Gosselin, P., \& Ladouceur, R. (2001). Intolerance of uncertainty and worry: Investigating specificity in a nonclinical sample. Cognitive Therapy and Research, 25, 551-558.

Fan, Q., \& Olatunji, B. O. (2013). Individual differences in disgust sensitivity and health-related avoidance: Examination of specific associations. Personality and Individual Differences, 55, 454-458.

Fergus, T. A. (2013). Cyberchondria and intolerance of uncertainty: Examining when individuals experience health anxiety in response to internet searches for medical information. Cyberpsychology, Behavior, and Social Networking, 16, 735-739.

Fetzner, M. G., Asmundson, G. J., Carey, C., Thibodeau, M. A., Brandt, C., Zvolensky, M. J., \& Carleton, R. N. (2014). How do elements of a reduced capacity to withstand uncertainty relate to the severity of health anxiety? Cognitive Behaviour Therapy, 43, 262-274.

Gangemi, A., Mancini, F., \& van den Hout, M. (2012). Behavior as information: If I avoid, then there must be danger. Journal of Behavior Therapy and Experimental Psychiatry, 43, 1032-1038.

Gerolimatos, L. A., \& Edelstein, B. A. (2012). Predictors of health anxiety among older and young adults. International Psychogeriatrics, 24, 1998-2008.

Haidt, J., McCauley, C., \& Rozin, P. (1994). Individual differences in sensitivity to disgust: A scale sampling seven domains of disgust elicitors. Personality and Individual Differences, 16, 701-713.

Henry, J. D., \& Crawford, J. R. (2005). The short-form version of the Depression Anxiety Stress Scales (DASS-21): Construct validity and normative data in a large non-clinical sample. British Journal of Clinical Psychology, 44, 227-239.

Hills, S. L., Russell, K., Hennessey, M., Williams, C., Oster, A. M., Fischer, M., \& Mead, P. (2016). Transmission of Zika virus through sexual contact with travelers to areas of ongoing
transmission-Continental United States, 2016. Morbidity and Mortality Weekly Report (MMWR), 65, pp. 215-216.

Krautwurst, S., Gerlach, A. L., Gomille, L., Hiller, W., \& Witthöff, M. (2014). Health anxiety-An indicator of higher interoceptive sensitivity? Journal of Behavior Therapy and Experimental Psychiatry, 45, 303-309.

Lau, J. T. F., Kim, J. H., Tsui, H. Y., \& Griffiths, S. (2008). Perceptions related to bird-to-human avian influenza, influenza vaccination, and use of face mask. Clinical and Epidemiological Study, 36, 434-443.

Liao, Q., Cowling, B. J., Lam, W. W. T., \& Fielding, R. (2011). The influence of social-cognitive factors on personal hygiene practices to protect against influenzas: Using modelling to compare Avian A/H5N1 and 2009 pandemic A/H1N1 influenzas in Hong Kong. International Journal of Behavioral Medicine, 18, 93-104.

Lovibond, S. H., \& Lovibond, P. F. (1995). Manual for the depression anxiety stress scales (2nd edn.). Sydney: Psychology Foundation.

McNeil, D. G. (2016). Obama administration to transfer Ebola funds to Zika fight. The New York Times, April 6 2016. Retrieved from http://www.nytimes.com/2016/04/07/health/zika-virus-budgetebola.html.

Meade, A. W., \& Craig, B. (2012). Identifying careless responses in survey data. Psychological Methods, 17, 437-455.

Niles, A. N., Dour, H. J., Stanton, A. L., Roy-Byrne, P. P., Stein, M. B., Sullivan, G., ... Craske, M. G. (2014). Anxiety and depressive symptoms and medical illness among adults with anxiety disorders. Journal of Psychosomatic Research, 78, 109-115.

Nunnally, J., \& Bernstein, I. (1994). Psychometric theory. New York: McGraw-Hill.

Olatunji, B. O. (2009). Incremental specificity of disgust propensity and sensitivity in the prediction of health anxiety dimensions. Journal of Behavior Therapy and Experimental Psychiatry, 40, 230-239.

Olatunji, B. O., Etzel, E. N., Tomarken, A. J., Ciesielski, B. G., \& Deacon, B. (2011). The effects of safety behaviors on health anxiety: An experimental investigation. Behaviour Research and Therapy, 49, 719-728. doi:10.1016/j.brat.2011.07.008.

Olatunji, B. O., \& Sawchuk, C. N. (2005). Disgust: Characteristic features, social implications, and clinical manifestations. Journal of Social and Clinical Psychology, 24, 932-962.

Olatunji, B. O., Williams, N. L., Tolin, D. F., Sawchuk, C. N., Abramowitz, J. S.., Lohr, J. M., et al. (2007). The disgust scale: Item analysis, factor structure, and suggestions for refinement. Psychological Assessment, 19, 281-297.

Reif, W., Hiller, W., \& Margraf, J. (1998). Cognitive aspects of hypochondriasis and the somatization syndrome. Journal of Abnormal Psychology, 107, 587-595.

Reiss, S., Peterson, R., Taylor, S., Schmidt, N., \& Weems, C. F. (2008). Anxiety sensitivity index consolidated user manual: ASI, ASI-3, and CASI. Columbus, OH: IDS Publishing Corporation.

Rosen, N. O., \& Knäuper, B. (2009). A little uncertainty goes a long way: State and trait differences in uncertainty interact to increase information seeking by also increases worry. Health Communication, 24, 228-238.

Salkovskis, P. M., Rimes, K. A., Warwick, H. M., \& Clark, D. M. (2002). The health anxiety inventory: Development and validation of scales for the measurement of health anxiety and hypochondriasis. Psychological Medicine, 32, 843-853.

Salkovskis, P. M., \& Warwick, H. M. (2001). Making sense of hypochondriasis: A cognitive model of health anxiety. In G. J. G. Asmundson, S. Taylor \& B. J. Cox (Eds.), Health anxiety: A Clinical and research perspectives on hypochondriasis and related conditions (pp. 46-64). New York, NY: Wiley.

Santora, M. (2016). As experts confront the hazards of Zika, fear and uncertainty hover. The New York Times. Retrieved from http://www.nytimes.com/2016/07/02/nyregion/ 
theyre-out-there-in-zika-health-officials-see-a-mystery-on-mosquito-wings.html?_r=0.

Schmidt, N. B., Lerew, D. R., \& Trakowski, J. H. (1997). Body vigilance in panic disorder: Evaluating attention to bodily perturbations. Journal of Consulting and Clinical Psychology, 65(2), 214-220.

Scott, K. M., Bruffaerts, R., Tsang, A., Ormel, J., Alonso, J., Angermeyer, M. C., ... Von Korff, M. (2007). Depression-anxiety relationships with chronic physical conditions: results from the World Mental Health Surveys. Journal of Affective Disorders, 103, 113-120.

Tausczik, Y., Faasse, K., Pennebaker, J. W., \& Petrie, K. J. (2012). Public anxiety and information seeking following the H1N1 outbreak: Blogs, newspaper articles, and Wikipedia visits. Health Communication, 27, 179-185.

Taylor, S., \& Asmundson, G. J. G. (2004). Treating health anxiety: A cognitive behavioral approach. New York: Guilford Press.

Taylor, S., McKay, D., \& Abramowitz, J. S. (2012). Hypochondriasis and health-related anxiety. In P. Sturney \& M. Hereson (Eds.), Handbook of evidence-based practice in clinical psychology, Volume II (pp. 603-619). New York: Wiley.

Taylor, S., Zvolensky, M., Cox, B., Deacon, B., Heimberg, R., Ledley, D. R., et al. (2007). Robust dimensions of anxiety sensitivity:
Development and initial validation of the Anxiety Sensitivity Index-3 (ASI-3). Psychological Assessment, 19, 176-188.

Tolin, D. F., Woods, C. M., \& Abramowitz, J. S. (2006). Disgust sensitivity and obsessive-compulsive symptoms in a non-clinical sample. Journal of Behavior Therapy and Experimental Psychiatry, 37, 30-40.

Warwick, H. M. C. (1989). A cognitive-behavioral approach to hypochondriasis and health anxiety. Journal of Psychosomatic Research, 3, 705-711.

Weck, F., Esch, S., \& Rohrmann, S. (2014). The role of disgust in patients with hypochondriasis. Anxiety, Stress, and Coping, 27, 576-586.

Wheaton, M. G., Abramowitz, J. S., Berman, N. C., Fabricant, L. E., \& Olatunji, B. O. (2012). Psychological predictors of anxiety in response to the H1N1 (swine flu) pandemic. Cognitive Therapy and Research, 36, 210-218.

World Health Organization (WHO) (2016). Situation report: Zika virus, microcephaly, Guillain-Barré syndrome. Retrieved from http://apps.who.int/iris/bitstream/10665/246112/1/zikasitrep23Jun2016-eng.pdf?ua=1.

Xie, X.-F., Stone, E., Zheng, R., \& Zhang, R.-G. (2011). The 'Typhoon Eye Effect': Determinants of distress during the SARS epidemic. Journal of Risk Research, 14, 1091-1107. 